\section{Über die Oxydation von Paraffin zu Wachs im ultravioletten Licht.}

Von AD. GRüN und TH. WIRTh.

(Mitteilung aus dem chemischen Laboratorium 1 der Firma Georg Schicht A.-G. in Aussig a. E.

(Eingeg. 31./8. 1920.)

Vor einiger Zeit haben wir an anderer Stelle eine Auswahl aus den Ergebnissen unserer Arbeiten über die Oxydation von Paraffin und Paraffinkohlenwasserstoffen mit Luftsauerstoff mitgeteilt ${ }^{1}$ ). Ein wesentliches Ergebnis unserer Untersuchung war die Feststellung, $\mathrm{da} B$ aus dem Paraffin unter geeigneten Reaktionsbedingungen vorwiegend Ester gebildet werden; neben Estern aus größtenteils hochmolekularen. Säuren und höheren Alkoholen enthalten die Reaktionsprodukte beträchtliche Mengen freier Säuren, ferner ein wenig unveränderte Kohlenwasserstoffe und andere unverseifbare Bestandteile, sie sind also Substanzgemische, die als Wa ch se bezeichnet werden müssen. Auch die Mischungsverhältnisse von Säuren und Alkoholen, freien und veresterten Säuren usw., sind bei diesen Produkten denen verschiedener tierischer und pflanzlicher Wachse ziemlich ähnlich, doch ist die Zusammensetzung anscheinend komplizierter, die Zahl der einzelnen Komponenten größer. Diese künstlichen Wachse gleichen in mancher Beziehung Mischungen verschiedenartiger natürlicher Wachse und lassen sich auch durch gewisse Lösungsmittel zum Teil soweit zerlegen, daß die härtesten jedoch plastischen - Fraktionen dem Bienenwachs, die weichsten Fraktionen hingegen gewissen flüssigen Tierwacbsen ähneln.

Die Oxydation von Paraffin zu Fettsäuren ist, unabhängig von uns, von verschiedenen Fachgenossen durchgeführt worden; in den zum Teil schon vor unserer oben erwähnten Mitteilung erschienenen Veröffentlichungen ${ }^{2}$ ) findet sich kein Hinweis darauf, da B man durch Oxydation des Paraffins direkt Ester, Wachse, erhalten kann. Hingegen ist die Darstellung von Estern neben Fettsäuren durch Oxydation von Paraffin im ultravioletten Licht schon in der amerikanischen Patentschrift Nr. 1158205 von Th. T. G r a y beschrieben. Obwohl diese Patentschrift bereits zur Zeit der Inangriff nahme unserer Versuche erschien (Erteilung am 26./10. 1915) blieb sie uns bis lange nach Abschluß derselben unbekannt - wir hatten, vermutlich durch den nichtssagenden Titel „Process of Treating Hydrocarbon-Oils" irregeführt, die Ankündigung des Patentes unbeachtet gelassen. Man kann nicht sagen, daß das praktisch wichtige Ergebnis unserer Arbeit durch das Verfahren von Gra y vorweg genommen wird; für unser Verfahren ist ja die rasche Durchführung der Oxydation unter $A$ us s o hlu $B$ irgendwelcher katalytischer Hilfsmittel, seien es Kontaktstoffe oder chemisch-aktive Strahlen, kennzeichnend. Die Ausführung der Oxydation ohne Bestrahlung des Reaktionsgemisches mit ultraviolettem Licht ist jedenfalls eine wesentliche Vereinfachung gegenüber dem Verfahren von G r a y und stellt somit einen technischen Fortschritt dar. Der Fortschritt ist um so größer, je geringer der durch die Verwendung des ultravioletten Lichtes etwa bedingte sogenannte "Mehreffekt" ist, sofern durch die Bestrahlung überhaupt ein Mehreffekt erzielt wird. Zur Klärung der Frage, ob - und gegebenenfalls in welchem $\mathrm{MaBe}$ - die Oxydation des Paraffins durch die Belichtung gefördert wird, haben wir eine Reihe von Parallelversuchen angestellt. Diese Versuche ergaben nun, daß - wenigstens unter den von uns eingehaltenen Pedingungen - die Reaktion durch ultraviolettes Licht überhaupt nicht beeinfluBt wird.

Versuchsanordnung: Als Reaktionsgefä $B$ diente ein Uviolglas. rohr von $36 \mathrm{~cm}$ Länge und $4,3 \mathrm{~cm}$ lichter Weite mit rundem Boden, in das mittels Kork ein Zuleitungs. und ein Ableitungsrohr, sowie ein Thermometer. eingeführt war. Die Luft wurde einer Bombe entnommen und durch einen Rotamesser $(R)$ zugeleitet, die $a b$ ziehenden Gase und Dämpfe traten in einen schräg aufsteigenden leeren Kühlermantel und aus diesem in mit Glasscherben gefüllte eisgekühlte Vorlagen. Das Reaktionsgefä $B$ wurde in einem Luftbad aus starker Asbestpappe $(12 \times 12 \times 40 \mathrm{~cm})$ montiert; eine Wand desselben war für die Bestrahlung der ganzen Länge nach etwa $4 \mathrm{~cm}$ breit ausgeschnitten, zwei andere gegenüberliegende Wände für die Beobachtung der Durchmischung und das Ablesen des Thermometers mit durch Glimmerplatten verschlossenen Fenstern versehen. Bei den Belichtungsversuchen wurde eine Uviollampe (110 Volt) so aufgestellt, daß ihre Leuchtröhre $(L)$ sich gerade vor dem Ausschnitt des Luftbades befand. Das Reaktionsgefä $\beta$ oder das in ihm aufgewirbelte Paraffinluftgemisch wurde also der ganzen Länge nach voll bestrahlt; jedenfalls war auch die Tiefenwirkung genügend, denn die Dicke der Paraffinschicht war ja im Ruhezustand nur $4,3 \mathrm{~cm}$, bei der intensiven Aufwirbelung sogar nur ein Bruchteil

1) Grün, Ulbrj ch u. Wirth, Ber. 53, 987 [1920].

2) M. B erg m a n n, Angew. Chem. 31. 69 [1918]; Fantowerke A.-G., ebenda, 115, 252; C. K e 1 b e r, Ber. 53, 66 [1920]; L. U b b e lo hde u. S. Eisenstein, Chem. Centralbl. 1920, IT, 22, H. Franck, Chem.Ztg. 44, 309 [1920]; F. Fis cher u. W Schneider, Ber. 53, 922 [1920].

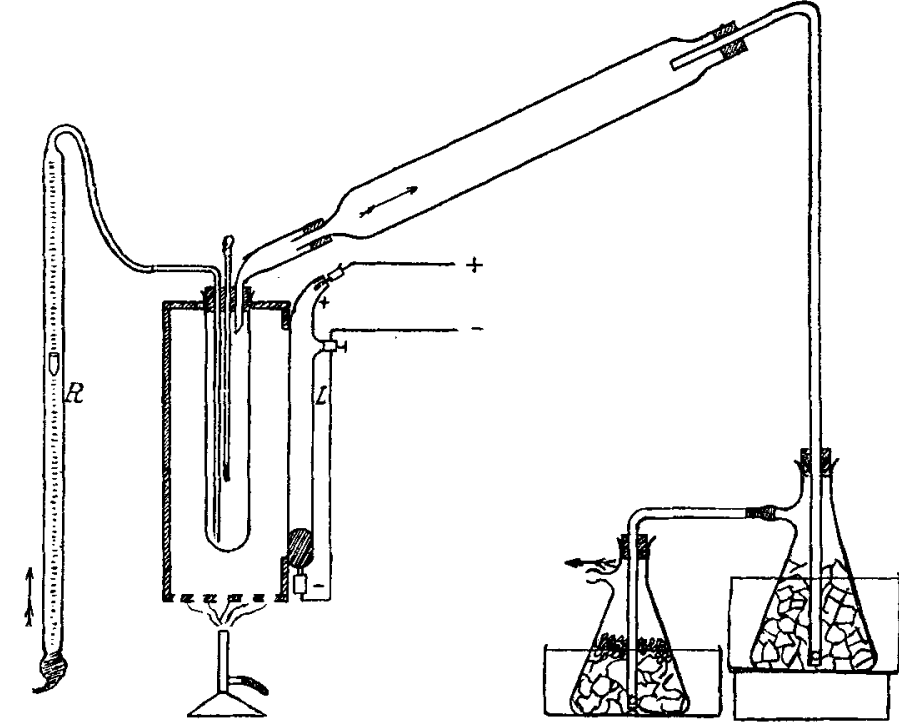

davon, während Gr a y eine Schicht von 3 Zoll (etwa $7^{1 / 2} \mathrm{~cm}$ ) Dicke aus einer Entfernung von 4 Zoll, allerdings mit einer 220.Voltlampe, belichtete.

Bei der Auswahl der Versuchsbedingungen wurde auf das erreichbare Maximum der Fettsäureausbeute zugunsten einer größeren Ausbeute an Estern verzichtet. Es wurden je $100 \mathrm{~g}$ Paraffin, E. P. $52^{\circ}$, auf $160^{\circ}$ angewärmt und ein Luftstrom von 101 in der Minute durchgeleitet. Die Einwirkungsdauer war bei zwei Parallelversuchen 3 Stunden, bei den übrigen $51 / 2$ Stunden. Selbstverständlich wurden die Bedingungen bei den zu vergleichenden Versuchen, je einer im Uviollicht und einer ohne. Belichtung, auf das genaueste gleichgehalten.

Schon beim Auswägen der Reaktionsprodukte zeigte sich völlige Übereinstimmung zwischen den Uviollicht- und den anderen Versuchen: bei den Versuchen von 3 stïndiger Dauer waren in beiden Fällen $86 \mathrm{~g}$ Substanz im Reaktionsgefäß geblieben, bei allen Versuchen von längerer Dauer rund $78 \mathrm{~g}$; die restlichen Substanzmengen fanden sich in den Vorlagen. Von den Rohprodukten wurden zu. nächst einige Kennzahlen bestimmt, die, in nachstehender Tabelle 1

Tabelle 1. Kennzahlen der Rohprodukte (Rückstände):

\begin{tabular}{c|c|c|c|c|c|c|}
\hline & & & Erst. P. & $\begin{array}{c}\text { Säure- } \\
\text { zahl }\end{array}$ & $\begin{array}{c}\text { Ester- } \\
\text { zahl }\end{array}$ & $\begin{array}{c}\text { Vers. } \\
\text { Zahl }\end{array}$ \\
\hline \hline 3 & Versuch 1 & Unbelichtet & $43,8^{\circ}$ & 49,0 & 81,8 & 130,8 \\
Stunden & Versuch 1a & Im Uviollicht & $44,5^{\circ}$ & 41,0 & 67,2 & 108,2 \\
$51 / 2$ & Versuch 2 & Unbelichtet & $42,1^{\circ}$ & 65,8 & 109,5 & 175,3 \\
Stunden & Versuch 2a & Im Uviollicht & $42,0^{\circ}$ & 67,3 & 114,0 & 181,3 \\
$51 / 2$ & Versuch 3 & Unbelichtet & $41,9^{\circ}$ & 67,7 & 127,0 & 194,7 \\
Stunden & Versuch 3a & Im Uviollicht & $42,2^{\circ}$ & 65,2 & 131,0 & 196,2
\end{tabular}

zusammengestellt sind. Die Kennzahlen stimmen verhältnismäßig, d. h. in Anbetracht der tumultuarisch und kompliziert verlaufenden Reaktionen, sehr gut überein. Die Abweichungen sprechen jedenfalls nicht zugunsten der Bestrahiung.

Auch die Kennzahlen der Destillate stimmen, wie ersichtlich, sehr gut überein, die Produkte sind praktisch kaum verschieden, das Destillat aus dem ohne Belichtung ausgeführten Versuch ist sogar, ebenso wie der Rückstand, ein wenig weiter oxydiert.

Tabelle 2. Kennzahlen von Destillaten:

\begin{tabular}{c|c|c|c|c}
\hline & Săurezahl & Esterzahl & Vers. Zahl \\
\hline Versuch 2 & Unbelichtet & 130,5 & 98,0 & 228,5 \\
Versuch 2a & Im Uviollicht & 116,8 & 103,5 & 220,3
\end{tabular}

Zur weiteren Untersuchung der Rohprodukte (Rückstände) wurde jedes für sich allein verseift, die unverseifbaren Bestandteile abgesehieden und die darauf in Freiheit gesetzten Fettsäuren in die in Wasser löslichen und unlöslichen getrennt. Von den unlöslichen Säurèn bestimmten wir Säurezahl, Verseifungs: und Esterzahl (diese von inneren Estern, Estoliden oder Semilactiden herrührend) bei den unverseifbaren Bestandteilen genügten zur Charakterisierung die Hydroxylzahlen. Die Ergebnisse der Analysen sind in nachI stehender Tabelle 3 geordnet. 
Tabelle 3. Zusammensetzung der Reaktionsprodukte.

\begin{tabular}{|c|c|c|c|c|c|c|c|c|}
\hline & & $\begin{array}{l}\text { Unlösliche } \\
\text { Säuren }\end{array}$ & $\begin{array}{l}\text { Lösliche } \\
\text { Säuren }\end{array}$ & $\begin{array}{c}\text { Un- } \\
\text { verseifbar }\end{array}$ & $\begin{array}{l}\text { S. Z. } \\
\text { der }\end{array}$ & $\begin{array}{l}\text { E. } Z \text {. } \\
\text { niösl. }\end{array}$ & $\begin{array}{l}\text { V. } \mathrm{Z} \text {. } \\
\text { uren }\end{array}$ & $\begin{array}{c}\text { Hydroxyl } \\
\text { zahl des } \\
\text { Unvers. }\end{array}$ \\
\hline $\begin{array}{l}\text { Versuch } 1 \\
\text { Versuch la }\end{array}$ & $\begin{array}{l}\text { Unbelichtet } \\
\text { Im Uviollicht }\end{array}$ & $\begin{array}{l}39,6 \% \\
34,4 \%\end{array}$ & $\begin{array}{l}6,1 \% \\
5,4 \%\end{array}$ & $\begin{array}{l}54,3 \% \\
60,2 \%\end{array}$ & $\begin{array}{l}180,0 \\
178,0\end{array}$ & $\begin{array}{l}33,7 \\
36,4\end{array}$ & $\begin{array}{l}213,7 \\
214,4\end{array}$ & $\begin{array}{l}85,6 \\
77,2\end{array}$ \\
\hline $\begin{array}{l}\text { Versuch } 2 \\
\text { Versuch } 2 a\end{array}$ & $\begin{array}{l}\text { Unbelichtet } \\
\text { Im Uviollicht }\end{array}$ & $\begin{array}{l}49,3 \% \\
49,8 \%\end{array}$ & $\begin{array}{l}8,4 \% \\
9,2 \%\end{array}$ & $\begin{array}{l}42,3 \% \\
41,0 \%\end{array}$ & $\begin{array}{l}188,3 \\
188,5\end{array}$ & $\begin{array}{l}41,3 \\
39,2\end{array}$ & $\begin{array}{l}229,6 \\
227,7\end{array}$ & $\begin{array}{l}101,5 \\
109,0\end{array}$ \\
\hline $\begin{array}{l}\text { Versuch } 3 \\
\text { Versuch } 3 \mathrm{a}\end{array}$ & $\begin{array}{l}\text { Unbelichtet } \\
\text { Im Uviollicht }\end{array}$ & $\begin{array}{l}52,9 \% \\
50,8 \%\end{array}$ & $\begin{array}{r}9,6 \% \\
10,6 \%\end{array}$ & $\begin{array}{l}37,5 \% \\
38,6 \%\end{array}$ & $\begin{array}{l}192,0 \\
184,0\end{array}$ & $\begin{array}{l}33,7 \\
32,9\end{array}$ & $\begin{array}{l}225,7 \\
216,9\end{array}$ & $\begin{array}{l}117,0 \\
110,5\end{array}$ \\
\hline
\end{tabular}

Die Zusammensetzung der Reaktionsprodukte, ihre Gehalte an Fettsäuren und an Alkoholen, sowie die Kennzahlen dieser Bestandteile, stehen mit den in der Tabelle 1 zusammengestellten Kennzahlen der Rohprodukte selbst im besten Einklang. Es bestätigt sich, daß bei den Parallelversuchen mit kürzerer Einwirkungsdauer die Oxydation ohne Belichtung sogar etwas weiter gegangen ist als die Oxydation unter Bestrahlung; die Ausbeute an unlöslichen Säuren ist um 5\%, größer, ebenso ist nach den Hydroxylzahlen der unverseifbaren Bestandteile die Ausbeute an Alkoholen größer. Die Zusammensetzung der Fettsäuren ist jedoch in beiden Fällen genau dieselbe, die Kennzahlen stimmen fast innerhalb der Fehler grenzen überein. Auch bei den Parallelversuchen 3 und 3 a blieb die unter Bestrahlung ausgeführte Oxydation ein klein wenig hinter der anderen zurück, doch erfolgte in diesem Falle weniger eine Vermehrung der Fettsäureausbente, als eine weitergehende Oxydation der Säuren selhst. - Auffällig ist, daß von den unter ganz gleichen Bedingungen ausgeführten vier Versuchen von $5 \%$ stündiger Dauer die beiden Parallelversuche 2 und 2 a etwas geringere Ausbeuten als 3 und 3 a gaben. Möglicherweise hängt dies damit zusammen, daß bei den letzteren Versuchen Luft aus einer neuen Bombe, vermutlich mit anderem Feuchtigkeitsgehalt, verwendet wurde.

Ts bedarf kaum einer Frwähnung, daB sich die Produkte der Parallelversuche auch bei der technischen Aufarbeitung als praktisch gleichwertig erwiesen. Fraktionierungen mittels Alkohol ergaben aus belichtetem und aus unbelichtetem Material gleiche Mengen harter, plastischer Wachse, deren Kennzahlen nur um wenige Einheiten voneinander abwichen.

Die Untersuchung ergab somit einwandfrei, daß das ultraviolette Licht auf die Oxydation des Paraffins überhaupt keinen Einfluß ausübte. - Wenn G r a y zu dem Ergebnis gelangte, daß die Be strahlung zur Erreichung des von uns erzielten technischen Effektes nicht nur nützlich, sondern sogar durchaus notwendig sei, so folgt daraus (die Richtigkeit seiner Beobachtungen vorausgesetzt) 1. Vermutlich hat $\mathrm{G} r$ a y nicht die richtigen, $d$. $h$. zur weitgehenden Oxydation ohne Belichtung erforderlichen Peaktionsbedingungen getroffen (die Angaben der Patentschrift sind zum mindesten nur ganz vage, z. B. wird bezüglich der Temperatur , vorzugsweise zwischen $100^{\circ}$ und $200^{\circ \prime \prime}$ (!) angegeben). 2. Unter solchen unzulinglichen Bedingungen könnte die Oxydation durch Bestrahlung gefördert werden, eine wenigstens teilweise Kompensation stattfinden. Aber sei dem wie immer, nachdem einmal gezeigt wurde, da $B$ die Umwandlung des Paraffins in Fettsäuren und deren Este bei Einhaltung gewisser Reaktionsbedingungen o h $\mathrm{n}$ e Bestrahlung mindestens ebensogut durchführbar ist, wie $u n t$ e $\mathbf{r}$ Bestrahlung, so ist diese eben vollkommen überflüssig. Es wird niemandem einfallen, Uviollicht oder dergleichen anzuwenden, was eine kompliziertere und kostspieligere Apparatur erfordert und auch die Betriebskosten wesentlich erhöht, wenn derselbe technische Effekt durch andere, einfachere Maßnahmen, wie etwa eine geringe Frhöhung der Temperatur, erzielt werden kann.

Aussig, im Angust 1920.

[A. 149.]

\section{Die Bedeutung der Magnesia als Düngemittel.}

\author{
Von Dr. A. JACOB, Berlin.
}

(Vortrag, gehalten auf der Hanptversammlung des Vereins deutscher Chemiker iu Hannover 1930 in der gemeinsamen Sitzung der Fachgruppen für anorganische Chemie, analytische Chemie, Kaliindustrie und organische Chemie.)

(Eingeg. am 23./9. 1920.)

Schon Lie big hat auf Grund von Aschenanalysen erkannt, daß die Magnesia ein unentbehrlicher Pflanzennährstoff ist. Die Wirkung einer künstlichen Zufuhr von Magnesiasalzen auf den Acker wurde zum ersten Male eingehender untersucht, als man die bei der
Düngung mit Kalirohsalzen anfänglich gelegentlich bemerkten Mißerfolge dem Chlor magnesiumgehalt dieser Salze zuschrieb; indes stellte sich heraus, da 3 diese schädigende Wirkung wohl nur dem hohen Chlorgehalte des Chlormagnesiums zuzuschreiben war. Erneut kam die Magnesiafrage auf die Tagesordnung im Anschluß an die L öw sche Hypothese vom Kalkfaktor, welche besagte, daß die Funktionen des Kalks und der Magnesia in der Pflanze auf das innigste miteinander verknïpft sind und daß es erforderlich sei, diese Nährstoffe den Pflanzen in einem für jede Pflanzengruppe besonders bestimmten Verhältnisse anzubieten. Die zahlreichen Untersuchungen, welche über diese Frage an. gestellt wurden, ergaben zwar keine Bestätigung für die von L ö w aufgestellte Theorie der Magnesiawirkung, andererseits zeigten sie aber doch, da.B unter gewissen Verhältnissen mit einer ertragsteigernden Wirkung einer Magnesiadüngung zu rechnen war. Ein neues Licht warf auf diese Frage die 1907 von Prof. $W$ ills tät ter gemachte Entdeckung, daB das Chlorophyll eine organische Magnesiumverbindung mit einem Gehalte von etwa $3 \%$ Magnesium ist. Die Anwesenheit des Magnesiums im Chlorophyll deutet darauf hin, daß dem Magnesium eine wichtige Rolle beim Hauptlebensprozesse der Pflanze, der Assimilation, zuzusprechen sei. Diese zunächst rein theoretische Annahme wurde gestützt durch die Beobachtung, daß die holländischen Landwirte seit Jahren eine ständig wachsende Vorliebe für die Verwendung von dem sog. Patentkali zeigten, einem Doppelsalze von schwefelsaurem Kali und schwefelsaurer Magnesia. Diese Gründe veranlaßten das Deutsche Kalisyndikat, der Magnesiafrage seine Aufmerksamkeit zuzuwenden. In den Jahren 1917-1919 wurden von der Agrikulturabteilung des Kalisyndikats daher zahlreiche Düngungsversuche angestellt, welche nachprüfen sollten, in welcher Weise ein Magnesiagehalt der Düngung auf den Ernteertrag einwirkt. Die Ergebnisse von 39 Kartoffelversuchen zeigten, $\operatorname{da} \beta$ man auf manchen Bodenarten mit einer fördernden Wirkung der Magnesiadüngung auf den Kartoffelertrag rechnen kann. Die durchschnittlichen Ergebnisse der Ernteerträge waren bei diesen Versuchen die folgenden :

$\begin{array}{cccc}\text { Kalifreie Grunddüngung mit Stickstoff und } & \begin{array}{c}\text { dz. Knollen } \\ \text { je ha }\end{array} & \begin{array}{c}\text { dz. Stärke } \\ \text { je ha }\end{array} \\ \text { Phosphorsäure . . . . . . . . . . . } & 202 & 37,1 \\ \text { Grunddüngung und Chlorkalium . . . . . } & 244 & 40,1 \\ \text { ", } & \text { und schwefelsaures Kali . } & 250 & 44,2 \\ \text { ", Chlorkalium und Kieserit . } & 253 & 41,5 \\ \text {, } & \text { und Kalimagnesia . . . . } & 253 & 44,0\end{array}$

Besonders erwiesen sich Sandböden und Moorböden für eine Zufuhr von schwefelsaurer Magnesia dankbar. Die mit der Ausführung der Magnesiadüngung zusammenhängenden Fragen werden augenblicklich erst noch der vollständigen Klärung durch wissenschaftliche Gefäßversuche entgegengeführt, welche an mehreren Versuchsstationen vorgenommen werden.

Um einer zu erwartenden starken Nachfrage der Landwirtschaft nach magnesiahaltigen Düngemitteln gerecht werden zu können, hat das Kalisyndikat jetzt bereits die Frage in Angriff genommen, in welcher Form ein geeignetes magnesiahaltiges Düngemittel herzustellen sei. Eine wesentliche Vergrößerung der Darstellung von schwefelsaurer Kalimagnesia würde nämlich erhebliche Abwässerschwierigkeiten mit sich bringen. Da die schwefelsaure Kalimagnesia ferner dasjenige Produkt der Kaliindustrie ist, in welchem die meisten veredelnden Arbeitsleistungen zu bezahlen sind, erscheint es geboten, dieses Salz in erster Linie für den Auslandsabsatz heranzuziehen, da uns daran liegen muß, dem Auslande möglichst keine Rohprodukte zu verkaufen, sondern Fertigfabrikate, in denen gleichzeitig die aufgewandte Arbeit mit bezahlt werden muß. Da bei den vorgenommenen Düngungsversuchen eine Mischung von Chlorkalium und Kieserit in ihrer Wirkung der schwefelsauren Kalimagnesia nur wenig nachgestanden hatte, wurde die Darstellung eines solchen Mischsalzes für den Bedarf der deutschen Landwirtschaft ins Auge gefaßt. Die vorläufig vorgenommenen Versuche über die Darstellungsmöglichkeit und die Lagerbeständigkeit eines solchen Mischsalzes lassen hoffen, daB der Magnesiabedarf der deutschen Landwirtschaft in dieser Form vorteilhaft wird befriedigt werden können. Für die Kalïndustrie wird sich damit gleichzeitig die Möglichkeit einer besseren Verwertung der großen Kieseritmengen ergeben, die bis jetzt zu einem großen Teile nur als wertloser Bergeversatz Verwendung finden konnten. 